\title{
Risk Factors and Molecular Study of Vimentin Gene (VIM), Associated with Female Breast Cancer in Khartoum, Sudan
}

\author{
Hala Abdalgader Khairalseed Abas ${ }^{1}$, Hind Abdelaziz Elnasri', \\ Mona Abdelrahman Mohmed Khaier, ${ }^{2, *}$ \\ ${ }^{1}$ Center of Medical Research, Faculty of Medicine, Al-Neelain University, Khartoum, Sudan \\ ${ }^{2}$ Department of Molecular Biology and Bioinformatics, College of Veterinary Medicine, University of Bahri, Khartoum, Sudan
}

Email address:

munakhaier@gmail.com (M. A. M. Khaier)

${ }^{*}$ Corresponding author

\section{To cite this article:}

Hala Abdalgader Khairalseed Abas, Hind Abdelaziz Elnasri, Mona Abdelrahman Mohmed Khaier. Risk Factors and Molecular Study of Vimentin Gene (VIM), Associated with Female Breast Cancer in Khartoum, Sudan. Cancer Research Journal. Vol. 9, No. 2, 2021 , pp. $92-97$. doi: $10.11648 /$ j.crj.20210902.12

Received: May 31, 2020; Accepted: July 4, 2020; Published: May 8, 2021

\begin{abstract}
Background: The aim of this study was to investigate the possible risk factors and mutations in VIM gene among Sudanese's breast cancer women in Khartoum State. Methods: This case-control study involved 45 patients with breast cancer and 45 controls. It was conducted across three hospitals and two laboratories in Khartoum State. A structural questionnaire was used to obtain data regarding age, family history, menarche, marriage, menopause, pregnancy, nulliparous and parous women, breast-feeding, use of fertility or contraceptive drugs and grade of the disease. DNA from patient and control tissues was extracted using extraction kits. PCR was conducted to amplify VIM gene using specific primers. PCR products were sequenced in order to detect the mutation in VIM gene. Data was analyzed using Pearson's Chi-square tests to identify risk factors associated with breast cancer. Results: The study showed that the main risk factors associated with breast cancer were family history with first degree relative, menarche, irregularity of menarche, reproductive factors such as pregnancy, breast-feeding and nulliparous. DNA sequencing revealed no mutations in VIM gene associated with breast cancer in Sudanese women in Khartoum State. Conclusion: The association of other risk factors such as menopausal status, age of menopausal, oral contraceptive birth control and fertility hormones needs more illumination and further work. Other genes associated with breast cancer can be investigated.
\end{abstract}

Keywords: Breast Cancer, Risk Factors, Sudan, VIM Gene

\section{Introduction}

Breast cancer remains the most common malignancy in women across the world [1]. Although the incidence rate is higher in Europe and the United States the death rates are insignificant [2]. The incidence of breast cancer varies from 89.7 per 100,000 in East Africa to 19.4 per 100,000 individuals in West Europe [3]. Although breast cancer incidence is lower in Sub-Saharan African countries than in developed countries, the cancer picture in Sub-Saharan Africa and especially in Sudan is changing. Lately, breast cancer incidence and mortality rates are increasing. It is the most common cancer in Sudanese women living in Khartoum State [4]. A high standard of mortality rates (ASR) for breast cancer in women living in Khartoum State -using the agestandardized rates per 100000 person-year between 1966 and 2000 , were 60.8 and 66.8 per 100000 , respectively. This data is higher than other studies reported in black women in East Africa, Harare, Zimbabwe (46.8 per 100,000, 2006- 2010) and in Kampala, Uganda (31.0 per 100,000, 1991-2006) [5]. In Sudan, Khartoum State, for the period from 2009-2010 the first data from National Population-based Cancer Registry (NCR) was established. The data revealed a total of 6771 incident cases of cancer, 3125 (46.2\%) were men and 3646 (53.8\%) were women [6].

Several epidemiological risk factors such as age, family history, menarche, pregnancy, nulliparous and porous women, menopause, Hormonal birth control, fertility drugs, breast feeding and marriage, different diagnosis stages of 
disease are considered as-significant factors for breast cancer development among women in Africa $[2,7,8]$.

Genetics plays a limited but important role as a risk factor for breast cancer. About 5\%-10\% of breast cancer cases demonstrate an autosomal dominant inheritance. Many researchers in breast cancer had identified specific genes that potentially confer a high risk of developing breast cancer such as BRCA1, BRCA2 and VIM gene [9].

$V I M$ gene has gained great importance as a canonical marker of epithelial-mesenchymal transition (EMT), also known to maintain cellular integrity and provide resistance against stress [10]. A variety of processes occurring in cells undergoing malignant transformation and metastasis involve VIM gene [11]. Evaluating the expression pattern of VIM gene in normal and cancer tissues can be of great value in tumor diagnosis and prognosis [12]. In Sudan there was scarce research about the mutations in VIM gene [13].

The aim of this study was to identify some risk factors and their association with developing breast cancer among Sudanese women such as age, family history, menarche, marriage, menopause, pregnancy, number of children, breastfeeding and using of fertility or contraceptivedrugsand which stage at diagnosis is common. In addition to investigating the mutations in exon one of VIM gene.

\section{Material and Methods}

\subsection{Study Area and Target Groups}

This was a Case Control study. An Ethical clearance was obtained from Ministry of Health - Khartoum. The study was conducted in different hospitals (Asia and Bahri Hospitals) and two laboratories (Ribat Hospital Laboratory and National Health laboratory). The study was carried during 2018-2019. The studied groups were 45 female with tumor as cases and 45 females with benign tumor or no malignancy seen in the breast as control. Both groups were matched on age ( \pm 3 years), diagnosis hospital (same hospital), and examination time (within 2 months). After receiving their consents for participation in the study, a structural questionnaire including age, family history, menarche, marriage, menopause, pregnancy, Nulliparous and parous women, breastfeeding, use of fertility or contraceptives (Hormonal birth control)drugs was done.

\subsection{Molecular Study}

Tissue samples for molecular analysis were obtained after positive (cases) or negative (controls) breast cancer confirmation. DNA was extracted using manufacturer Kit (itaq-iNtRon biotechnology. Korea). The isolated DNA was quantified using Nano drop spectrophotometer and gel electrophoresis.

\subsubsection{Primer Design for VIM Gene}

The researcher designed primers (using primer-3 software):

-Forward5'-GGCTCAGATTCAGGAACAGC- 3'.

-Reverse primer 5'- AGCCTCAGAGAGGTCAGCAA - 3'.

\subsubsection{Polymerase Chain Reaction (PCR)}

DNA was added to Maxime PCR Premix kit (i-taq-iNtRon biotechnology. Korea) with $1 \mu \mathrm{L}$ forward primer and $1 \mu \mathrm{L}$ reverse primer. The total volume was adjusted to $20 \mu \mathrm{L}$ using deionized water. A control reaction containing sterile deionized water, but without template DNA was always performed to confirm the absence of contamination. Amplification conditions were set as, A denaturing step at $94^{\circ} \mathrm{C}$ for $2 \mathrm{~min}, 40$ cycles of: denaturation $\left(94^{\circ} \mathrm{C}\right.$ for $\left.20 \mathrm{sec}\right)$, annealing $\left(58^{\circ} \mathrm{C}\right.$ for $\left.30 \mathrm{sec}\right)$ and extension $\left(72^{\circ} \mathrm{C}\right.$ for $\left.1 \mathrm{~min}\right)$, and with a final extension step at $72^{\circ} \mathrm{C}$ for $7 \mathrm{~min}$.

\subsection{Data Analysis}

\subsubsection{Statistical Analysis}

Statistical package of social science (SPSS) version 21 was used for analysis of the data to study the relationship between variables and breast cancers among the two groups.

\subsubsection{In Silico Analysis}

The PCR products for each sample were gel purified prior to sequencing analysis. Sequencing of PCR was performed using automated sequencer (Macrogen Co. Lt., Seoul, Korea). Quality control of the nucleotides chromatogram was done using Finch TV software version 1.4.0 to ensure that all ambiguous sites are correctly called and determine the overall quality [14]. Then nucleotides sequences were searched for sequence similarity using nucleotide BLAST. Highly similar sequences were retrieved from NCBI and subjected to multiple sequence alignment using the BioEdit software [15].

\section{Results}

Ninety women were enrolled in this study, 45 with breast cancer considered as cases and 45 women who were negatively diagnosed were selected as control.

\subsection{Demographic Data}

Data regarding different factors such as age, family history, menopausal status, age at menopause, use of fertility or contraceptives (Hormonal birth control)drugs and stage of breast cancer were investigated. Most cases (73.3\%) were above 40 years old (Figure 1) and (66.7\%) had a family history of breast cancer. About $46.6 \%$ had a first degree relative diagnosed with breast cancer (Figure 2).

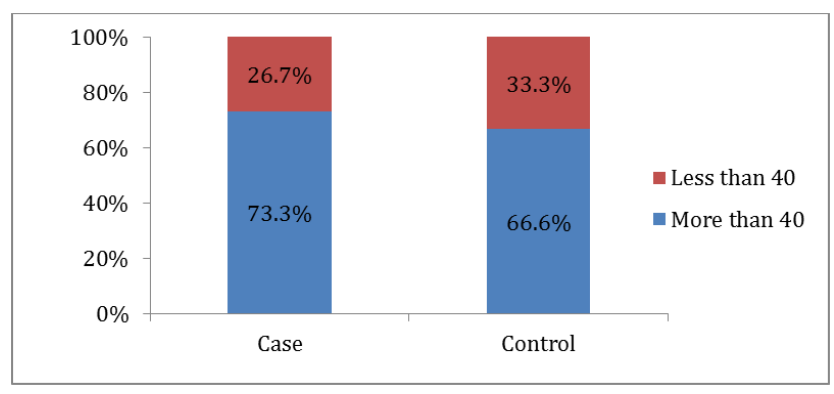

Figure 1. Classification according to Age in Control and Case Groups. 


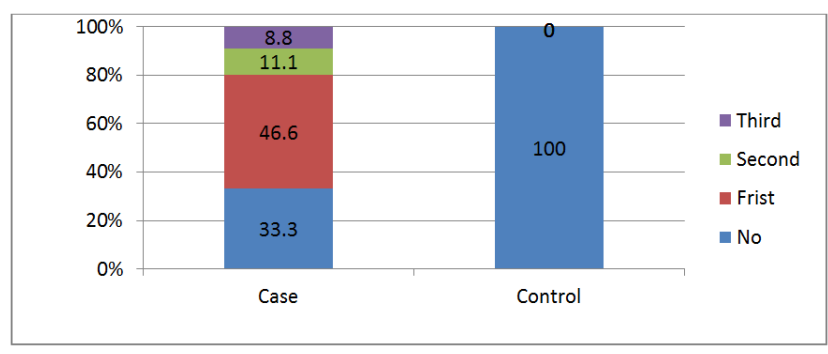

Figure 2. The Degree of Kinship within Family.

The patients were at different stages of cancer as shown in Figure 3

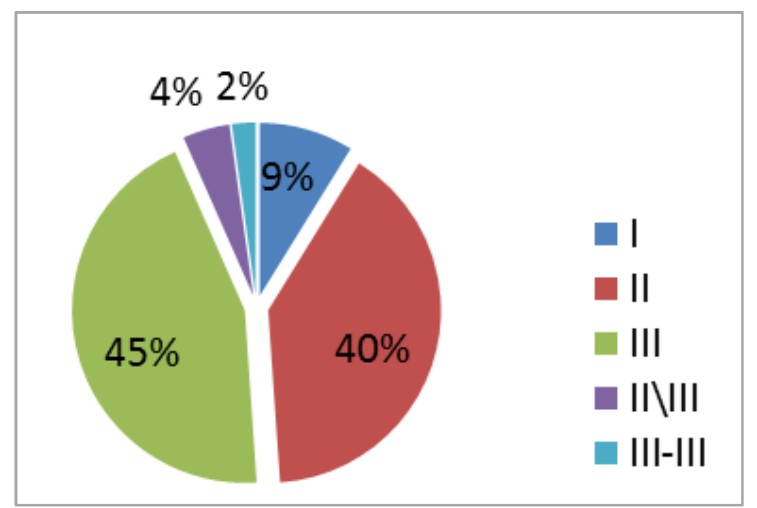

Figure 3. Classification of patients according to different stages of cancer.

The data obtained regarding the various questions asked in questionnaire are shown in Table 1.

Table 1. Data regarding different risk associated factors.

\begin{tabular}{lll}
\hline Parameter & Case & Control \\
\hline A) Age of First Menarche & & \\
1- Less than 14 years & $31.1 \%$ & $73.3 \%$ \\
2- More than 14 years & $68.8 \%$ & $26.6 \%$ \\
B) Regularity of Menarche & & \\
1- Yes & $26.6 \%$ & $62.2 \%$ \\
2-No & $73.3 \%$ & $37.7 \%$ \\
C) Marital Status & & \\
1- Yes & $42.2 \%$ & $82.2 \%$ \\
2-No & $57.7 \%$ & $17.7 \%$ \\
D) Age of marriage & & \\
1- Less than 18 years & $24.4 \%$ & $73.3 \%$ \\
2- More than 18 years & $17.7 \%$ & $8.8 \%$ \\
3- unmarried & $57.7 \%$ & $17.7 \%$ \\
E) Menopause Status & & \\
1- Yes & $42.2 \%$ & $37.7 \%$ \\
2-No & $57.7 \%$ & $62.2 \%$ \\
F) Age of Menopause (years) & & \\
1- Non & $51.1 \%$ & $62.2 \%$ \\
2-35-44 & $11.1 \%$ & $17.7 \%$ \\
3- 45- 54 & $28.1 \%$ & $20 \%$ \\
4- more than 55 & $8.8 \%$ & 0 \\
G) Pregnancy & & \\
1- Married but No pregnancy occurred & $22.2 \%$ & $8.8 \%$ \\
2- Yes & $20 \%$ & $71 \%$ \\
3- Unmarried & $57.7 \%$ & $22.2 \%$ \\
H) Age of pregnancy & & \\
1-Unmarried & $57.7 \%$ & $22.2 \%$ \\
2- No pregnancy with marriage & $22.2 \%$ & $4.4 \%$ \\
3-Less than 18 & $6.6 \%$ & $64.4 \%$ \\
4-More than 18 & $13.3 \%$ & 8.8 \\
\hline & & \\
\hline
\end{tabular}

\begin{tabular}{lll}
\hline Parameter & Case & Control \\
\hline I) Number of Children & & \\
1-Unmarried & $55.5 \%$ & $22.2 \%$ \\
2- 1-5 & $17.7 \%$ & $55.5 \%$ \\
3- 6-10 & $4.4 \%$ & $20 \%$ \\
4- Married with no children & $22.2 \%$ & $2.2 \%$ \\
J) Breast feeding & & \\
1-Non applicable & $11.1 \%$ & $11.1 \%$ \\
2- Yes & $8.8 \%$ & $64.4 \%$ \\
3-No & $80 \%$ & $24.4 \%$ \\
K) Drugs used & & \\
1- None & $66.6 \%$ & $46.6 \%$ \\
2- Fertility drugs & $4.4 \%$ & $22.2 \%$ \\
3- Contraceptives (hormonal birth control) & $28.8 \%$ & $31.1 \%$ \\
\hline
\end{tabular}

\subsection{Association Between Different Risk Factors and Breast} Cancer

A significant association $(\mathrm{P}<0.00)$ was found between family history and breast cancer. The results among the breast cancer patients revealed that $46 \%$ had a first degree relative with the disease.

In addition, women with older age at menarche $(>14$ years old) were found to be at higher risk $(\mathrm{P}<0.00)$ for breast cancer $(68 \%)$ than women with younger age of menarche $(<14$ years old). The regularity of the menarche $33(73 \%)$ was also considered as a significant risk factor $(\mathrm{P}<0.0 .01)$. Unmarried women demonstrated a higher risk of breast cancer than others $(\mathrm{P}<0.00)$.

Another important risk factor was the age of marriage and age of pregnancy. Women married and got pregnant at younger age $(<18$ years old $)$ were found to be at lower risk $(\mathrm{P}<0.00)$ than women married at older age ( $>18$ years old). There was a significant association between breast cancer and breastfeeding $(\mathrm{P}<0.00)$ were $80 \%$ of the cases were not breast feeding. Furthermore, women with more children (parous women) were found to be at lower risk to develop breast cancer compared with women without children (Null-parous women) $(\mathrm{P}<0.00)$. The results showed most of the cases in this population were diagnosis at late stage $45 \%$ (III) $40 \%$ (II).

\subsection{Analysis of Molecular Results}

Following extraction and conducting PCR using the specific primers, the results of vimentin gene gave a band size of $\sim 172 \mathrm{bp}$ (Figure 4).

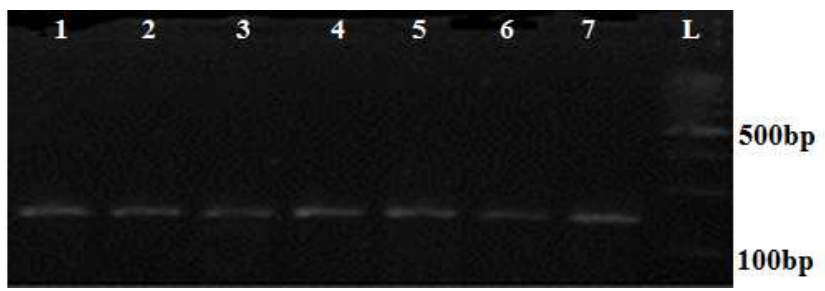

Figure 4. Agarose gel electrophoresis of amplified part of VIM gene.

Lane L: 100bp molecular ladder, Lane 1-6: Positive VIM gene $~ 172 b p$

No mutations in Vimentin (VIM) gene among Sudanese breast cancer women was identified after sequencing. The data showed $100 \%$ identity to VIM gene sequence retrieved 
from GenBank. (Figure 5)

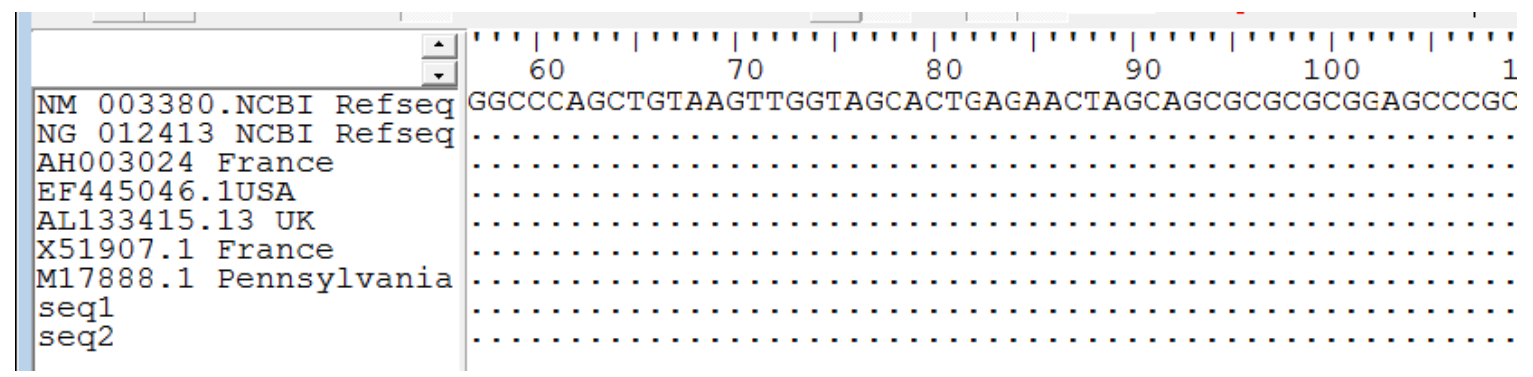

Figure 5. DNA Sequences Alignment of VIM Gene for Samples with reference sequences from NCBI Database, with 100\% Identity using BioEdit software.

\section{Discussion}

This study was a case-control study involving 90 Sudanese women living in Khartoum state conducted during the period 2018- 2019. In total, $50 \%$ of the cases were diagnosed with breast cancer and $50 \%$ were chosen as control with no malignancy seen.

In this study $(42.2 \%)$ of the patients were found to be in a postmenopausal age ( $>40$ years old) This is in agreement with Mohamed et al., who stated that the breast cancer is higher among postmenopausal women [16]. This observation disagreed with the higher incidence of breast cancer in younger women observed in 52 of 196 black American women (26\%) vs. 48 of 300 American cases [17]. Similarly, in a British study conducted to examine data collected from 445 patients newly diagnosed with breast cancer showed that black British patients were significantly younger $(p=0.001)$ with median age of 46 compared with 67 for the white patients with breast cancer [18].

In this study, family history of breast cancer was found to be a significant factor for developing the disease. This observation is similar to the study conducted in north east China showing that familial risks have a largely genetic basis on breast cancer [19].

This study also compared between first, second and third degree of relation of family relative and found strong significant association between females with cancer with a first-degree family member. This is similar to a study in Sweden which suggested that familial cancers had early onset mainly in individuals whose parents were affected [20].

Despite the early menarche (age below 14 years) which was not a significant risk factor for breast cancer among the studied population, epidemiological studies in Germany have a contradictory results which showed a decrease of breast cancer risk by later age at menarche. Generally, the risk decreased by $10-24 \%$ with each year of delay in menarche [21]

This study also revealed that most of the cases were unmarried or married after 18 years of age. This group had a higher risk for breast cancer when compared with married women especially before 18 years old. These results disagree with study done in the eastern region of Saudi Arabia, where it was observed that most breast cancer cases were married under 18years of age compared to the control group [22]. Since there is no previous study in this context in
Sudan, the late marriage will be perceptible risk factor of getting breast cancer in the future in Sudan.

Reproductive factors, like pregnancy and breastfeeding have been reported to have a protective role against breast cancer [23]. In Moroccan women there was clear significant relation between with full-term pregnancy and breast cancer compared with non-pregnant women [24].

Nulliparous women had a higher risk for breast cancer compared to porous women in this population. A metaanalysis of large Scandinavian epidemiological studies found that nulliparous women have a $30 \%$ increased risk compared with women who have had at least 1 full-term pregnancy [24].

According to results of the present study, breast feeding is of a protective factor against breast cancer in Sudanese women. These findings are consistent with a large collaborative studies in India which perceived that women who were breastfeeding for longer time have a lower risk by $4.3 \%$ for every 12 months of breastfeeding [25]. The clear explanation of this finding is the delay of the return of regular ovulatory cycles and decrease endogenous sex hormone levels [25].

Another possible explanation relates to structural changes that occur in the breast following lactation and weaning, meaning induced mammary differentiation which confers protective effects against breast cancer, also differentiated cells are comparatively more resistant to be transformed into cancerous cells [25].

The majority $(88.8 \%)$ of women presented in this population were diagnosed at stage III that was similar to the proportion of women with late-stage of breast cancer (III) from $50 \%$ to $27 \%$ in white women, and from $60 \%$ to $32 \%$ in black women at in the USA between 1973 and 2011 [26]. Four South African studies examined racial differences, which consistently showed a higher percentage of late-stage of breast cancer in black Africans (range 74-91\%) than white Africans (30-44\%) [26]. These findings may be attributable to the fact that patients ignore symptoms and do not access medical care at an early stage. In addition, this might due to a lack of education, a skeptical attitude towards western medicine, cultural customs that exclude and isolate diseased individuals [26].

Most of cases were not at menopausal status so the effect had no clear significant in this population. A study in USA carried during 2004 revealed that menopause will increase the risk of breast cancer [8]. 
The use of contraceptives (hormonal birth control) and oral fertility drugs had no clear significance association with breast cancer in this study. These result have the same view with Dutch study that showed women currently taking combined oral contraceptives have slight effect to devolved disease compared to never users, but there is no evidence of an increase in the risk ( 10 years after stopping the use of drugs) [27]. It disagrees with epidemiological studies from the Women's Health Initiative (WHI) which had a randomized trial and provided convincing evidence that the effect of oral contraceptives or hormone replacement therapy (HRT) increases breast cancer risk, but depending on strongly of hormonal constituents, duration of HRT use and time of cessation [27].

This study was conducted to investigate mutations in exon one of VIM gene and breast cancer in women. No mutations were found in exon one of VIM gene. The study of Noh et al in Maryland explained how VIM had been linked to the malignant transformation and metastatic spread of cancer cells [28].

Other observations of the structural of similarities between VIM and the proto-oncogenes c-fos, c-jun, Raf and the v-mos oncogene indicated vimentin-dependent control of gene expression can promote transformation of primary cells into malignant cancer cells [29].

Two reports showed that over expression of VIM protein is correlated with breast cancer and increased invasive behavior and promotion of migration of mammary epithelial cells [2930].

Vimentin-associated migration in pre-malignant breast cancer cells has been shown to be induced by H-Ras-V12G and Slug. Interestingly, the presence of VIM during Epithelial-Mesenchymal Transition (EMT) leads to up regulation of the receptor tyrosine kinase Axl that enhances the migratory behavior of breast epithelial cells [31].

Korschinget al., (2005) used immunohistochemistry technique for VIM protein study and 15 other differentiation markers in invasive breast cancer tissue samples, and concluded that neither EMT nor myoepithelial histogenesis could fully explain the origin of the vimentin-expressing cells in the tissues [29].

\section{Conclusion}

This study has revealed that family history with first degree relative, menarche, irregular of menarche, reproductive factors such as pregnancy, breast-feeding and nulliparous are the strongest risk factors associated with breast cancer for Sudanese women. No mutations in exon one of VIM gene among Sudanese breast cancer women were found (viewing $100 \%$ identity to VIM gene retrieved from GenBank).

\section{Recommendations}

Other risk factors playing an important role in breast cancer such as menopausal status, age of menopausal, contraceptive (hormonal birth control) and oral fertility drugs needs illumination and further work to show their association with breast cancer. It is recommended to increase the number of samples, RT-PCR for VIM gene expression in breast cancer women in Sudan and whole exons sequencing (exome) of VIM gene.

Also it is advisable to increase family awareness and knowledge about early marriage, pregnancy, natural breastfeeding.

\section{Acknowledgements}

Thanks and gratitude to all those who participated in this work. Special thanks to Dr. Alya Ahmed Salman, Assistant Professor of Pathology Department, Faculty of Medicine, AlNeelain University for her overall supervision. Thanks are also was extended to Department of Molecular Biology and Bioinformatics, College of Veterinary Medicine, University of Bahri were the practical was conducted.

\section{References}

[1] Susan L and Paul C. (1998); Epidemiology in practice: CaseControl Studies. Community Eye Health. 11 (28): 57-58.

[2] Siegel RL, Miller KD and Jemal A. (2015): Cancer statistics. CA Cancer J Clin; (65): 5-29.

[3] Fitzmaurice C, Dicker D, Pain A, Hamavid H, Moradi-Lakeh M, MacIntyre MF. (2013). The global burden of cancer. JAMA Oncol. 2015; (1): 505-527.

[4] Bray Fand Jemal A, Grey N. (2012). Global cancer transitions according to the Human Development Index (2008-2030): A population-based study. Lancet Oncol; (13): 790-801.

[5] Jamison, D. T., J. G. Breman, A. R. Measham, G. Alleyne, M. Claeson, D. B. Evans (2006). Disease control priorities in developing countries. World Bank, Washington, DC.

[6] Intisar E. Saeed, Hsin-Yi Weng, Kamal H Mohamed \& Sulma I. Mohammed. (2014). Cancer incidence in Khartoum, Sudan: first results from the Cancer Registry, 2009-2010. Cancer Medicine; 3 (4): 1075-1084.

[7] American Cancer Society. Breast Cancer Facts \& Figures 2017-2018. Atlanta: American Cancer Society, Inc. 2017.

[8] Stacey A. Missmer, A. Heather Eliassen, Robert L. Barbieri, Susan E. Hankinson (2004). Endogenous Estrogen, Androgen and Progesterone Concentrations and Breast Cancer Risk Among Postmenopausal Women, JNCI; 96 (24); 1856-1865.

[9] Lip worth L, Bailey LR, Trichopoulos D. (2000) History of Breast-Feeding in Relation to Breast Cancer Risk: a Review of the Epidemiologic Literature. J Natl Cancer Inst; 92 (11): 302-312.

[10] Jatoi I, Anderson WF, Rao SR, Devesa SS. (2005) Breast cancer trends among black and white women in the United States. J. Clin Oncol; 23 (31): 7836-7841.

[11] Cheng F, Eriksson J. E. (2017) Intermediate Filaments and the Regulation of Cell Motility during Regeneration and Wound Healing. Cold Spring Harb. Perspect. Biol; (9) 220-46. 
[12] Dauphin M, Barbe C, Lemaire S, Nawrocki-Raby B, Lagonotte E, Delepine G, Birembaut P, Gilles C, Polette M. (2013). Vimentin expression predicts the occurrence of metastases in non small cell lung carcinomas. Lung Cancer; 81: 117-122.

[13] Satelli A. and Li S. (. 2011). Vimentin in cancer and its potential as a molecular target for cancer therapy. Cell Mol Life Sci; 68 (18): 3033-3046.

[14] Http \www.finchTV.

[15] TA Hall - Nucleic acids symposium series (1999) jwbrown.mbio.ncsu.edu.

[16] Mohamed E. M. Saeed1, Jingming Cao1, Babikir Fadul, Onat Kadioglu, Hassan E. Khalid, Zahir Yassin, Siddig M. Mustafa, Elfatih Saeed And Thomas Efferth (2016) A Five-Year Survey Of Cancer Prevalence In Sudan. Anticancer Research; (36): 279-286.

[17] Jatoi I, Anderson WF, Rao SR, Devesa SS (2005). Breast cancer trends among black and white women in the United States. J Clin Oncol; 23 (31): 7836-7841.

[18] Bowen RL, Duffy SW, Ryan DA, Hart IR, Jones JL.(2008) Early onset of breast cancer in a group of British black women. Br J Cancer; 98 (2): 277-281.

[19] Yu ZG, Jia CX, Geng CZ, Tang JH, Zhang J, Liu LY. (2012) Risk factors related to female breast cancer in regions of Northeast China: a 1:3 matched case-control population-based study. Chin Med J (Engl); 125: 733-40.

[20] Kharazmi E, Fallah M, Sundquist K, Hemminki K. (2012) Familial risk of early and late onset cancer: nationwide prospective cohort study. BMJ; 345 (10): 1136-8076.

[21] Alexander K and Andrea SP. (2009) Established and Suspected Risk Factors in Breast Cancer Etiology. Breast Care; 4 (2): 82-87.

[22] Ibrahim G. Alghamdi ab, Issam I. Hussaina, Mohamed S. Alghamdic, Mohamed A. El-Sheemyd.(2015) Early marriage is a potential risk factor for female breast cancer in the Eastern
Region of Saudi Arabia. American Journal of Research Communication; 3 (7).

[23] Aizer AA, Chen M-H, McCarthy EP. (2013) Marital status and survival in patients with cancer. J Clin Oncol; (31): 386976.

[24] Britt K, Ashworth A, Smalley M. (2007) Pregnancy and the risk of breast cancer. Endocrine-Related Cancer; (14): 907-33.

[25] Babita NK, Singh M, Malik JS. (2014)Breastfeeding reduces breast cancer risk: a case-control study in north India. Int. $J$ Prev. Med; 791 (5).

[26] Elima Jedy-Agba, Valerie McCormack, Clement Adebamowo and Isabel dos-Santos-Silva. (2016)Stage at diagnosis of breast cancer in sub-Saharan Africa: a systematic review and meta-analysis. Lancet Glob Health; 4 (12): e923-e935.

[27] Collaborative Group on Hormonal Factors in Breast Cancer. Menarche, menopause, and breast cancer risk: individual participant meta-analysis, including 118,964 women with breast cancer from 117 epidemiological studies. Lancet Oncol. 2012; (13): 1141-1151.

[28] Noh H, Yan J, Hong S, Kong LY, Gabrusiewicz, K, Xia X, Heimberger A. B, Li S. (2016)Discovery of cell surface vimentin targeting $\mathrm{mAb}$ for direct disruption of GBM tumor initiating cells. Onco. Target; (7): 72021-72032.

[29] Kokkinos MI, Wafai R, Wong MK, Newgreen DF, Thompson EW, Waltham M. (2007)Vimentin and epithelial-mesenchymal transition in human breast cancer--observations in vitro and in vivo. Cells Tissues Organs; 185: 191-203.

[30] GillesC, Polette M, Zahm J. M, Tournier J. M, Volders L, Foidart J. M, Birembaut P. (1999)Vimentin contributes to human mammary epithelial cell migration. J. Cell Sci.; 112 (24): 4615-4625.

[31] Lahat G, Zhu Q S, Huang K L, Wang S, Bolshakov S, Liu J, Torres K, Langley RR, Lazar AJ, Hung M C.(2010) Vimentin Is a Novel Anti-Cancer Therapeutic Target; Insights from In Vitro and In Vivo Mice Xenograft Studies. PLoS ONE; (5): 101-105. 